\title{
LA-SUB--95-11
}

\section{STOCHASTIC ANALYSIS OF TRANSPORT OF CONSERVATIVE SOLUTES IN CAISSON EXPERIMENTS}

\author{
by
}

Gedeon Dagan

To be submitted to Los Alamos National Laboratory

\section{DISCLAIMER}

This report was prepared as an account of work sponsored by an agency of the United States Government. Neither the United States Government nor any agency thereof, nor any of their employees, makes any warranty, express or implied, or assumes any legal liability or responsibility for the accuracy, completeness, or usefulness of any information, apparatus, product, or process disclosed, or represents that its use would not infringe privately owned rights. Reference herein to any specific commercial product, process, or service by trade name, trademark, manufacturer, or otherwise does not necessarily constitute or imply its endorsement, recommendation, or favoring by the United States Government or any agency thereof. The views and opinions of authors expressed herein do not necessarily state or reflect those of the United States Government or any agency thereof. 


\section{DISCLAIMER}

Portions of this document may be illegible in electronic image products. Images are produced from the best available original document. 


\section{Introduction}

The Los Alamos National Laboratory has conducted in the past a series of experiments of transport of conservative and reactive solutes. The experimental setup and the experimental results are presented in a series of reports ${ }^{1,2,3,4}$. The main aim of the experiments was to validate models of transport of solutes in unsaturated flow at the caisson intermediate scale, which is much larger than the one pertaining to laboratory columns. First attempts to analyze the experimental results were by one-dimesnsional convective-dispersion models. These models could not explain the observed solute breakthrough curves and particularly the large solute dispersion in the caisson effluent Since there were some question marks about the uniformity of water distribution at the caisson top, the transport experiments were repeated under conditions of saturated flow5. In these experiments constant heads were applied at the top and the bottom of the caisson and the number of concentration monitoring stations was quadrupled. The analysis of the measurements by the same one-dimensional model indicated clearly that the fitted dispersivity is much larger than the pore-scale dispersivity and that it grows wiht the distance in an approximately linear fashion. This led to the conclusion, raised before, that transport in the caisson is dominated by heterogeneity effects, i.e. by spatial variability of the material. Such effects cannot be captured by traditional one-dimensional models.

In order to account for the effect of heterogeneity, the saturated flow experiments have been analyzed by using stochastic transport modeling6.7. The apparent linear growth of dispersivity with distance ${ }^{5,697}$ suggested that the system behaves like a stratified one ${ }^{8}$. Consequently, the model of Dagan and Bresler ${ }^{\theta}$ has been adopted in order to interpret concentration measurements. In this simple model the caisson is viewed as a bundle of columns of different permeabilities, which are characterized by a p.d.f. (probability denasity function). From a physical standpoint, the presence of such a structure can be explained as a result of the nonuniform packing of the caisson material, for instance due to different compactions in the center and near the walls. By using the model and by assuming convective transport, the expected value $\langle M(z, t)\rangle$, of the cumulative solute mass which has passed through the caisson cross-section at depth $z$ and time $t$, was evaluated. By adopting a lognormal permeability distribution, an analytical expression of $\langle\mathrm{M}\rangle$, depending on the logconductivity variance was derived. Since direct measurements of conductivity were not available, an inverse procedure was applied. First, the experimental $M(z, t)$ was inferred by interpolating among concentration

monitoring stations and integrating in space. Secondly, an ergodic assumption was adopted, namely that the entire population of conductivites is present in the single realization of the caisson such that $M \simeq(M)$. Last, the value of the variance was identified by a best fit between 
measured $M$ and computed $\langle M\rangle$. Confidence in the model stemmed from its ability to reproduce quite accurately the curves of $M$ as function of $t$ at different depths.

The present study addresses two issues related to the caisson experiments. The first one is that of uncertainty of $M$, the mass arrival function, in the saturated experiments. The ergodic assumption mentioned above presumes that there is no such uncertainty. From a theoretical standpoint ${ }^{8}$ this is correct only if the caisson cross-sectional area is infinite, cr more precisely if the ratio between the diameter $d$ and the conductivity correlation scale $I$ tends to infinity. In practice the variance of $M$ becomes very small if the ratio is finite but sufficiently large. The purpose of this part (Section 2) is to examine the dependence of $\mathrm{CV}_{M}$, the coefficient of variation of $M$, upon $d / I$. Since conductivity measurements were not available, this ratio was not known. Hence, the purpose of the computation at this stage is to estimate the needed magnitude of $d / I$ to ensure that $C V_{M}$ is sufficiently small, to allow for the exchange between $M$ and $(M)$. Furthermore, the results are indicative of the heterogeneous structure existing in the caisson. The second issue (Section 3) is the analysis of the caisson unsaturated experiments with the conservative solute ${ }^{1,2}$. In this analysis we evaluate again the expected value of the mass arrival (M) by using the same basic columnar model ${ }^{9}$, but adapted to the nature of the unsaturated flow and to the boundary condition of uniformly applied flux. Adopting again the ergodic hypothesis, a best fit is achieved between the observed $M$ in the effluent and the calculated $(M)$ for a certain value of the variance of the logvelocity. By using a model of the flow, this value is related to the saturated conductivity variance, which was determined previously. Again, the uncertainty of $M$ is examined by relating its variance to the ratio $d / I$, between the caisson diameter and the velocity correlation scale.

Finally, in Sect. 4 we present a few conclusions drawn from the developments of the present study, as well as recommendations for future caisson experiments.

\section{Uncertainty of mass arrival in saturated flow experiments}

Let $z$ be a downward vertical Cartesian coordinate, with origin at the caisson top and $x, y$ coordinates in the horizontal plane. In line with our previous analysis ${ }^{67}$ the hydraulic conductivity $\mathrm{K}_{\mathrm{s}}$ is regarded as a function of $\mathrm{x}, \mathrm{y}$ solely. With bounadry conditions of constant head at $z=0$ and $z=L$ (caisson bottom), the exact solution of the flow problem is 


$$
q(x, y)=K_{s}(x, y) J
$$

where $q$ is the vertical specific discharge and $J$ is the head gradient. Indeed, (2.1) expresses Darcy's Law and also satisfies exactly the continuity equation. Assuming a constant effective porosity we have

$$
\mathrm{u}(\mathrm{x}, \mathrm{y})=\frac{\mathrm{q}}{\mathrm{n}}=\frac{\mathrm{J}}{\mathrm{n}} \mathrm{K}_{\mathrm{s}}
$$

where $\mathrm{u}$ is the velocity and $\mathrm{n}$ the effective porosity.

A slug of solute of concentration $C_{0}$ is inserted during a time period $\Delta t$ and at time $t=0$ in the inlet reservoir. The solute is convected by the fluid through the caisson and the resident concentration $\mathrm{C}$ is given by

$$
C(x, y, z, t)=C_{0} \Delta t \operatorname{n~} u(x, y) \delta(z-u t)
$$

where $A$ is the caisson cross-sectional area and $\delta$ is the Dirac operator. Eq. (2.3) expresses the assumption of pure convection with neglect of pore-scale dispersion. This is justified by the smallness of the pore-scale dispersivity in comparison with the heterogeneity scale. However, (2.3) can be easily generalized to include pore-scale dispersion by replacing the concentrated pulse in (2.3) by a Gaussian one. Our computations show clearly that with the values of porescale dispersivity derived from measurements, the effect is negligible and does not warrant the additional computational burden.

The solute mass flux $Q_{C}$ through the caisson cross-section is

$$
Q_{C}(z, t)=n \iint_{A} C(x, y, z, t) u(x, y) d x d y
$$

and the cumulative solute mass which has crossed the cross-section is given by 


$$
\begin{gathered}
M(z, t)=\int_{0}^{t} Q_{C}\left(z, t^{\prime}\right) d t^{\prime}= \\
C_{0} \Delta t n \iint_{A} \int_{0}^{t} u^{2} \delta\left(z-u t^{\prime}\right) d t^{\prime} d x d y=C_{0} \Delta t n \iint_{A} u H(u-z / t) d x d y
\end{gathered}
$$

where $H(z)$ is the Heaviside step function, i.e. $H=1$ for $\nabla>0$ and $H=0$ for $z<0$. $M$ is the basic quantity we are using in order to analyze transport. The reason is that we rely mainly on the measurement of the concentration of the caisson effluent, which is flux averaged, i.e. the product of the water discharge $Q$ times the effluent concentration is precisely $Q_{C}$.

We regard $\mathrm{K}_{\mathrm{s}}$ and the resulting $\mathrm{u}(2.2)$ as random functions, to reflect their irregular spatial variability and the uncertainty affecting their distributions. In line with the literature ${ }^{8}$, we assume $\mathrm{K}$ to be lognormal and operate with $\nu=\operatorname{lnu}$ as independent variable. Hence, the univariate p.d.f. $f(\nu)$ is given by

$$
\mathrm{f}(\nu)=\frac{1}{(2 \pi)^{1 / 2} \sigma_{\nu}} \exp \left[-\frac{\left(\nu-\mathrm{m}_{\nu}\right)^{2}}{2 \sigma_{\nu^{2}}}\right]
$$

The joint p.d.f. $f\left(\nu_{1}, \nu_{2}\right)$ of $\nu_{1}=\nu\left(x_{1}, y_{1}\right)$ and $\nu_{2}=\nu_{2}\left(x_{2}, y_{2}\right)$ is binormal and of correlation coefficient $\rho_{\nu}$, ie. ${ }^{10}$

$$
\left.f\left(\nu_{1}, \nu_{2}\right)=f\left(\nu_{1}\right) f\left(\nu_{2}-\rho_{\nu} \nu_{1}\right) /\left(1-\rho_{\nu^{2}}\right)^{1 / 2}\right]
$$

The expected value of $M(2.5)$ is obtained by substituting first $u=\exp (\nu)$, multiplying by $f(\nu)$ (2.6) and integrating from $-\infty$ to $+\infty$. The result is

$$
\begin{gathered}
\langle\mathrm{M}(\mathrm{z}, \mathrm{t})\rangle=\mathrm{C}_{0} \Delta \operatorname{tnA} \frac{\langle\mathrm{u}\rangle}{2}\left[1+\operatorname{erf}\left(\frac{\ln \tau+\sigma_{\nu}^{2 / 2}}{(2)^{1 / 2} \sigma_{\nu}}\right]\right. \text { where } \\
\langle\mathrm{u}\rangle=\exp \left(\mathrm{m}_{\nu}+\sigma_{\nu}{ }^{2 / 2}\right) \text { and } \tau=\mathrm{t}(\mathrm{u}\rangle / \mathrm{z}
\end{gathered}
$$


This result has been obtained previously ${ }^{6,7}$ and has been employed in order to identify $\sigma_{\nu}^{2}$ from the concentration measurements, as explained above in Section 1. Since the solute has been inserted as a pulse of duration $T=6$ days, $(M)(2.8)$ was integrated once more over time. It has been found ${ }^{6,7}$, however, that except for the smallest $z$, approximating the pulse by a concentrated one at midT is an accurate approximation of $\langle M\rangle$ for the actual pulse provided that $\Delta t$ is replaced by $T$ in (2.8). Hence, we shall emplpy in the sequel the expression of (M) (2.8). Furthermore, we rewrote it in a dimensionless form as follows

$$
\frac{(M)}{M_{0}}=\frac{1}{2}\left[1+\operatorname{erf}\left(\frac{\ln \tau+\sigma_{\nu}^{2} / 2}{(2)^{1 / 2} \sigma_{\nu}}\right]\right.
$$

where $M_{0}=C_{0} T n\langle u\rangle$ is the expected value of the total solute mass in the caisson.

We have assumed previously6, that $M$ is ergodic, i.e. that $\langle M\rangle$ can be exchaged with $M$ in any realization and particularly in the caisson experiment. This was the justification of the identification preocedure in which we have taken $\langle u\rangle$ equal to its measured value and $(M) / M_{0}$ the same.

We are going now to examine this assumption by computing the variance $\sigma_{\mathrm{M}}^{2}$ and the coefficient of variation $C V M=\sigma_{M} /(M)$. Only if the latter is much smaller than unity is the ergodic assumption bound to hold. Toward this aim we write $M^{2}(2.5)$ and take its expected value as follows

$$
\left\langle M^{2}(z, t)\right\rangle=M_{0}^{2} \frac{1}{A^{2}} \iint_{A} \iint_{A}\left\langle u_{1} u_{2} H\left(u_{1}-\frac{z}{t}\right) H\left(u_{2}-\frac{z}{t}\right)>d x_{1} d y_{1} d x_{2} d y_{2}\right.
$$

where $\mathrm{u}_{1}=\mathrm{u}\left(\mathrm{x}_{1}, \mathrm{y}_{1}\right)$ and $\mathrm{u}_{2}=\mathrm{u}\left(\mathrm{x}_{2}, \mathrm{y}_{2}\right)$ are velocities at two arbitrary points in the caisson crosssection. The integrands in (2.10) can be computed by substituting $u_{1}=\exp \left(\nu_{1}\right)$ and $u_{2}=\exp \left(\nu_{2}\right)$, respectively, multiplying by $f\left(\nu_{1}, \nu_{2}\right)(2.7)$ and integrating from $-\infty$ to $+\infty$. One quadrature can be carried out in a close form leading to

$$
u_{1} u_{2} H\left(u_{1}-\frac{z}{t}\right) H\left(u_{2}-\frac{z}{t}\right)>=
$$




$$
\begin{gathered}
\frac{\exp \left(2 m_{\nu}\right)}{4 \pi} \int_{a}^{\infty} \exp \left[\left(\sigma_{\nu}^{2}\left(1-\rho_{\nu}^{2}\right)-\lambda^{2}\right) / 2+\sigma_{\nu} \rho_{\nu} \lambda\right]\left\{1+\operatorname{erf}\left[\frac{\rho_{\nu} \lambda-a+\sigma_{\nu}^{2}\left(1-\rho_{\nu}^{2}\right)}{2^{1 / 2}\left(1-\rho_{\nu}^{2}\right)^{1 / 2}}\right]\right\} d \lambda \\
\text { with } a=\left(\sigma_{\nu}^{2} / 2-\ln \tau\right) / \sigma_{\nu}
\end{gathered}
$$

We have now to integrate (2.11) twice over the caisson area in (2.10). We assume that $K_{s}$ and $\nu$ are stationary and isotropic, i.e. the correlation coefficient $\rho_{\nu}$ depends on the distance $r=$ $\left[\left(x_{1}-x_{2}\right)^{2}+\left(y_{1}-y_{2}\right)^{2}\right]^{1 / 2}$ solely. Hence, the function (2.11) depends on $r$ through $\rho_{\nu}$. Then, we may reduce the quadruple integration in (2.10) to a sigle one by using Cauchy's algorithm ${ }^{8}$ and rewrite $\left\langle M^{2}\right\rangle(2.10)$ as follows

$$
\left\langle M^{2}\right\rangle=M_{0}^{2} \frac{4}{A} \int_{0}^{d} B(r / d)<u_{1} u_{2} H\left(u_{1}-\frac{z}{t}\right) H\left(u_{2}-\frac{z}{t}\right)>r d \lambda d r
$$

where $d$ is the caisson diameter, $A=\pi d^{2} / 4$ is its area and $B=\cos ^{-1}(r / d)-(r / d)\left(1-r^{2} / d^{2}\right)^{1 / 2}$ results from Cauchy's algorithm ${ }^{8}$.

To effectively evaluate $\left\langle M^{2}\right\rangle$ we have assumed an exponential $\rho_{\nu}=\exp (-r / M)$. Then, $\left\langle M^{2}\right\rangle$ can be calculated by a double integration, one over $\lambda$ and an additional one over $r$. The coefficient of variation $C V_{M}=\left(\left(M^{2}\right\rangle /\langle M)^{2}-1\right)^{1 / 2}$ results from this operation as a function of the dimensionless parameters $\sigma_{\nu}^{2}$ and $d^{\prime}=d / I$ and of the dimensionless time $T$ (2.8).

In the case in which $\mathrm{K}_{\mathrm{S}}$ is measured at a sufficiently large number of points in the caisson, the stationarity of the logconductivity and the value of the integral scale I can be determined by statistical inferrence. Since such measurements are not available, we have computed $\mathrm{CV}_{\mathrm{M}}$ for the value $\sigma_{\nu}=1.2$ identified previously6r, and for different ratios $\mathrm{d}^{\prime}$ between the caisson diameter and the logconductivity integral scale in the horizontal plane. The computation has been carried out numerically by carrying out the two quadratures in (2.12). The resulting $\mathrm{CV}_{\mathrm{M}}$ as function of $\tau=t\langle u\rangle / z$ is represented in Fig. 1 for a few $d^{\prime}$. In Fig. 2 we have represented $\langle M\rangle / M_{0}$ as function of $\tau$ as well as bounding curves at $95 \%$ interval of confidence. It is seen that a considerable reduction of the uncertainty of $M / M_{0}$ is achieved for $d^{\prime}=100$, i.e. for $\mathrm{I} \sim 3 \mathrm{~cm}$.

From the structure of $\left\langle M^{2}\right\rangle(2.12)$ and the associated $C V_{M}$ it can be seen that $C V_{M}$ is proportional to $1 / A$ i.e. to $d^{-2}$ for $d^{\prime} \gg 1$. This is proved by Fig. 3 in which the curves of Fig. 1 
practically collapse in one curve after multiplying $C V_{M}$ by $d^{\prime 2}$. It is emphasized that for a three-dimensional heterogeneous structure the drop of $\mathrm{CV}_{\mathbf{M}}$ with $\mathrm{d}^{\prime}$ is more rapid. Hence, the present results may be viewed as upper bounds of the variance of $M$.

\section{Analysis of transport in caisson unsaturated flow experiments}

(i) Introduction.

Unlike the saturated flow experiments, in the unsaturated ones the solute breakthrough curves were measured only at a few stations ${ }^{1,2,3}$. Consequently, we were not able to carry out an investigation similar to the one performed for saturated flow6r7. The presence of one sampling station at each depth does not permit one to compute, by interpolation, the curves of mass arrival as function of time. Hence, in the stochastic framework, the measured breakthrough curves can be viewed as samplings of the solute transport in a few streamtubes and they can serve to determine the magnitude of the pore-scale dispersion coefficient. The heterogeneity effect could be assessed, however, from the measurement of the concentration of the effluent which averages the contribution of the entire caisson volume to transport. It is precisely the large discrepancy between the pore-scale effect manifesting in the breakthrough curves and the large rate of spreading apparent in the outlet concentration that prompted previous studies ${ }^{4}$ to propose heterogeneity as the main dispersion mechanism.

Encouraged by the results obtained in the analysis of the saturated flow experiments ${ }^{6,7}$, we have decided to apply the model of Dagan and Bresler ${ }^{9}$ to the unsaturated flow case as well. A major difference between the two configurations is that while (2.1) is an exact solution of the flow equations for constant head boundary conditions, there is no such simple solution for unsaturated flow. Indeed, the simple extension of the flow model to unsaturated conditions is to assume that flow is gravitational and of constant vertical specific discharge $q$, imposed by the boundary condition at the caisson top. This leads, however, to the existence of gradients of the moisture content $\theta$ in the horizontal plane and in transverse components of the specific discharge vector, contradicting the model of vertical flow. Hence, a more accurate and complete analysis of the flow requires regarding it as three-dimensional. We can assume, however, that the horizontal gradients are weak as they are proportional to matric potential differences divided by the heterogeneity correlation scale in the horizontal plane. Since the caisson depth is only twice its diameter, it is reasonable to assume that the horizontal flow is of limited extent and regard flow as vertical and gravitaional. This picture can be validated by measuring the moisture content on 
a dense grid and breakthrough curves at a large number of stations. Although there are a relatively large number of $\theta$ measuring stations, they do not cover either the caisson area and the neighborhood of the walls in a comprehensive manner. Thus, validation of the model is a matter of future investigations (see Sect. 4). On the other hand, we may use the information gained from the analysis of the saturated experiments, namely the magnitude of the saturated logconductivity variance and check its compatibility with the results of the unsaturated flow transport.

(ii) Analysis of experimental data.

We have analyzed the two experiments of transport of iodide through the caisson summarized in report ${ }^{3}$ by Polzer et al. We have started by determining the actual water discharge through the caisson based on the effluent outflow measurements. Based on the Table of p. $103^{3}$, the cumulative oufflow is represented in Figs. 4 and 5. The day 77 corresponds to Dec. 6, 1984 whereas the day 150 is Febr. 17, 1985. It is seen from Figs. 4 and 5 that the actual flow through the caisson was quite steady, though small fluctuations were present. The average rates of flow, i.e. the average slopes in Figs. 4 and 5, were determined to be

$$
Q_{1}=\frac{20.6 \times 10^{3}}{149-77}=286 \mathrm{l} / \mathrm{day} ; \mathrm{Q}_{2}=\frac{21.4 \times 10^{3}}{216-149}=319 \mathrm{l} / \mathrm{day}
$$

respectively. The average specific discharges were, therefore,

$$
\bar{q}_{1}=Q_{1} / A=4.05 \mathrm{~cm} / \text { day } ; \bar{q}_{2}=Q_{2} / A=4.51 \mathrm{~cm} / \text { day }
$$

Our next task was to determine the cumulative solute mass $M(L, t)$, arriving at the bottom section at $\mathrm{z}=\mathrm{L}$. This has been done by multiplying the daily measured iodide concentration of the Table of p. $166^{3}$ by the corresponding daily outflow of the Table at page $103^{3} . M_{1}$ and $M_{2}$ for the two experiments are represented in Figs. 6 and 7, respectively. It is seen that the total solute mass in the two experiments was quite different, reflecting the difference in the concentrations at the inlet ${ }^{3}$. We have rendered the cumulative mass dimensionless by dividing it by $M_{0}$, the total mass in each experiment. The curves $M_{1}(L, t) / M_{0}$ and $M_{2}(L, t) / M_{0}$ are represented in Fig. 8. The time origin was shifted to day 77 and day 141, the beginning of the solute insert at $z=0$ in the two experiments. The striking result is the closeness of the two curves, demonstrating the 
repetibility of the experiment in spite of the difference in total mass and the slight one in the velocities. This result for $M(L, t) / M_{0}$ in Fig. 8 was the basis of our interpretative effort.

Next, we have analyzed the moisture content measurements $\theta$ of the Table of $p .100^{3}$. We have used the data at six depths and ten times. From the various computations we reproduce here the results for the average $\theta$ over each experiment period and over the entire caisson. We have also determined the variance of the $\ln \theta$ and the results are

$$
\theta_{1}=0.278 ; \quad \theta_{2}=0.29 ; \quad \sigma_{\ln \theta}^{2}=0.0046
$$

While the variance is not regarded as representative, since the cross-section was not covered in a comprehensive manner, the average values for the two experiments were taken as acceptable in view of the small variability of $\theta$.

Based on (3.2) and (3.3) we could determine the average pore-water velocity during the two experiments as follows

$$
\bar{u}_{1}=\bar{q}_{1} / \theta_{1}=14.6 \mathrm{~cm} / \text { day } \quad ; \quad \bar{u}_{2}=\bar{q}_{2} / \theta_{2}=15.6 \mathrm{~cm} / \mathrm{day}
$$

and the difference between the two velocities was narrowed down in comparison with that of the specific discharges.

(iii) The flow model.

We have adopted the Brooks and Corey ${ }^{11}$ relationship between unsaturated conductivity $\mathrm{K}$ and moisture content

$$
\mathrm{K} / \mathrm{K}_{\mathrm{S}}=\mathrm{S}^{\alpha} \simeq\left(\theta / \theta_{\mathrm{S}}\right)^{\alpha}
$$

where $S=\left(\theta-\theta_{\mathrm{ir}}\right) /\left(\theta_{\mathrm{S}}-\theta_{\mathrm{ir}}\right)$ is the relative saturation and $\alpha$ is a constant. We have neglected $\theta_{\mathrm{ir}}$, the irreducible saturation, since we did not have reliable data about it (it is poorly defined anyhow). Furthermore, for the relatively large saturations of (3.3), it is bound to play a minor role in (3.5). The coefficient $\alpha$ was assumed to be constant in the caisson, though field measurements indicate that $\alpha$ is spatially variable. Again, the available data were not sufficient to determine the variability of $\alpha$ in the caisson and we adopted the value derived for samples of crushed Bandelier tuff ${ }^{12}$, namely $\alpha=8.75$. 
The saturated conductivity $\mathrm{K}_{\mathrm{S}}$ and the saturated moisture content $\theta_{\mathrm{S}}$ are also interdependent, i.e. one can write

$$
\mathrm{K}_{\mathrm{s}}=\mathrm{k} \theta_{\mathrm{s}}^{\beta}
$$

where $\mathrm{k}$ is a constant. Eq. (3.6) is known as the Kozeny-Carman relationship and in absence of measurements for the caisson tuff, we have adopted the value in the literature ${ }^{13}$ of $\beta=3.5$.

We assume the flow in the caisson to be gravitational, in line with the basic model ${ }^{9}$. Hence, according to Darcy's law

$$
\mathrm{q}=\mathrm{K}
$$

Unlike the saturated experiments, the water was applied in the caisson uniformly at the top. As a matter of fact the distribution was not uniform in the upper part, as reflected by the measured breakthrough curves at the upper station ${ }^{3}$. However, since we are analyzing the effluent we disregard this effect, which adds to the other neglected sources of variability. We therefore assume that in each of the two experiments $q$ was constant and equal to the values of (3.2), respectively.

Finally, the pore velocity $u$ is given by

$$
u(x, y)=q / \theta(x, y)
$$

since in our model ${ }^{\ominus}$ the caisson is viewed as a bundle of columns of $K_{s}$ varying in the horizontal plane.

The Eqs. (3.5-3.8) summarize our simple flow model. It is seen that the spatial variability of the water velocity originates from that of $\theta$ solely. Taking the logarithm of $u, \xi=\ln u$, we obtain from $(3.5-3.8)$

$$
\xi=a+b \mu ; a=\frac{\beta-1}{\beta}\left(\operatorname{lnq}+\frac{\operatorname{lnk}}{\alpha-1}\right) ; b=\frac{1}{\beta} \frac{\alpha-\beta}{\alpha-1} ; \mu=\ln \mathrm{K}_{\mathrm{s}}
$$

In Eq. (3.9) $a$ and $b$ are constants, whereas the heterogeneity of the caisson medium manifexts in the spatial variability of $\mathrm{K}_{\mathrm{s}}$ and of its logarithm $\mu$. These have been determined in the saturated flow experiments ${ }^{6,7}$ (see also Sect. 2 here). In our model ${ }^{9} \mathrm{~K}_{\mathrm{S}}$ is a function of $\mathrm{x}, \mathrm{y}$ 
solely. Since we view $\mathrm{K}_{\mathrm{S}}$ as a random variable it is immediately seen that the variance of $\xi$ is related to that of $\mu$ by the simple relationship

$$
\sigma_{\xi}^{2}=b^{2} \sigma_{\mu}^{2}
$$

with $b$ given in terms of known constants by (3.9) and with $\sigma_{\mu}=\sigma_{\nu}=1.2$, the latter being the value identified from the saturated flow experiments ${ }^{6 \pi}$ (see Sect. 2).

(iv) The transport model.

The transport model ${ }^{9}$ follows closely the one of saturated flow,7 (Sect. 2).The resident concentration is given (see Eq. 2.3) by

$$
C(x, y, z, t)=C_{0} \Delta t q \delta(z-u t)
$$

where $C_{0}$ is the solute concentration in the fluid sprinkled on the caisson top, $\Delta t=T=6$ days is the pulse duration, $q$ is the constant specific discharge and $u$ is the variable pore velocity. This is different from (2.3) in which $q$ (2.1) was also variable. This, of course, reflects the different boundary conditions in the two cases.

We now have for the solute flux and cumulative mass through the caisson cross-section

$$
\begin{gathered}
Q_{C}(z, t)=q \iint_{A}^{t} C(x, y, z, t) d x d y \\
M(z, t)=\int Q_{C}\left(z, t^{\prime}\right) d t^{\prime}= \\
C_{0} \Delta t q \iint_{A} \int_{0}^{t} \delta\left(z-u t^{\prime}\right) d t^{\prime} d x d y=C_{0} \Delta t q \iint_{A} H\left(u-\frac{z}{t}\right) d x d y
\end{gathered}
$$


similarly to $(2.4,2.5)$. Assuming that $\xi=\operatorname{lnu}$ is normal of variance $\sigma_{\xi}^{z}$ (see Eq. 2.6), computations similar to those of Sect. 2, Eq. (2.9), lead to the following results for the expected value of the cumulative mass

$$
\frac{(M)}{M_{0}}=\frac{1}{2}\left[1+\operatorname{erf}\left(\frac{\ln \tau-\sigma_{\xi}^{2 / 2}}{(2)^{1 / 2} \sigma_{\xi}}\right]\right.
$$

where $\langle u\rangle=\exp \left(m_{\xi}+\sigma_{\xi}{ }^{2 / 2}\right)$ and $\tau=t\langle u\rangle / z$, precisely as in (2.8).

By the same token, assuming that $\xi$ at two different points in the caisson cross-section are bivariate normal (Eq. 2.7), computations similar to those of Sect. 2 lead to

$$
\begin{gathered}
\left\langle M^{2}(z, t)\right\rangle=M_{0}^{2} \frac{1}{A^{2}} \iint_{A} \iint_{A}\left\langle H\left(u_{1}-\frac{z}{t}\right) H\left(u_{2}-\frac{z}{t}\right)>d x_{1} d y_{1} d x_{2} d y_{2}\right. \\
\left\langle H\left(u_{1}-\frac{z}{t}\right) H\left(u_{2}-\frac{z}{t}\right)>=\frac{1}{(8 \pi)^{1 / 2}} \int_{a} \exp \left[-\frac{\mu^{2}}{2}\right]\left\{1+\operatorname{erf}\left[\frac{\rho_{\xi} \lambda-a}{\left.2^{1 / 2}(1-\rho)^{2}\right)^{1 / 2}}\right] d \lambda\right.\right.
\end{gathered}
$$

where a is the same as in (2.11) whereas $\rho_{\xi}=\rho_{\mu}$ is the correlation coefficient of logvelocity, and therefore of $\operatorname{lnK}_{s}$, at two points. The final result for $\left\langle M^{2}(z, t)\right\rangle$ is give now by

$$
\left(M^{2}\right)=M_{0}^{2} \frac{4}{A} \int_{0}^{d} B(r / d)\left\langle H\left(u_{1}-\frac{z}{t}\right) H\left(u_{2}-\frac{z}{t}\right)>r d \lambda d r\right.
$$

where B (see expression after 2.12) results from Cauchy algorithm. Eqs. (3.16) and (3.13) render

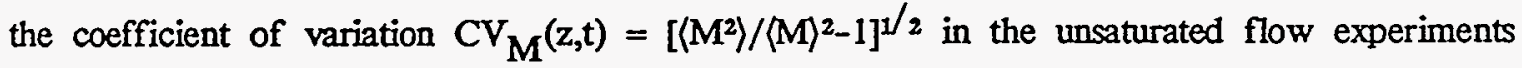
interms of two quadratures over $\lambda$ and $r$ respectively.

(v) Comparison with measured mass arrival at the outlet. 
Following our methodology67t for satuarated flow experiments (Sect. 2), we assume ergodicity and exchange the computed expected value of $(M) / M_{0}$ (3.13) with the one measured in the affluent (Fig. 8). By moving the origin in Fig. 8 by $T / 2=3$ days (the midpoint of the pulse application) and taking $\mathrm{z}=\mathrm{L}=564 \mathrm{~cm}$ for the depth of the outlet ${ }^{6,7}$ we have identified by a leastsquare procedure the best values of $\langle u\rangle$ and $\sigma_{\xi}$ which minimize the difference between the computed (3.13) and the measured (M)/M (Fig. 9). The best-fitted theoretical and measured curves are represented together in Fig. 9. The value of the squared difference between the curves was quite low and equal to 0.018 . The resulting optimal values were

$$
\langle\mathrm{u}\rangle=15.9 \mathrm{~cm} / \text { day } ; \quad \sigma_{\xi}^{z}=0.08
$$

Since direct measurements of the conductivity were not available, the validity of our model can be assessed by (i) comparing ( $u\rangle$ (3.17) with the measured averages (3.4) and (ii) by comparing $\sigma_{\xi}$ (3.17) with (3.10) based on the saturated flow experiments. It is seen that $\langle u\rangle(3.17)$ is somewhat larger than the average $u=15.1 \mathrm{~cm} /$ day. The somputed value of $\sigma_{\xi}(3.10)$ with $\alpha=8.75, \beta=3.5$ and $\sigma_{\mu}=1.2$ (the latter from the saturated flow analysis ${ }^{6,7}$ ) is $\sigma_{\xi}=0.194$ whereas the optimal one (3.17) is $\sigma_{\xi}=0.283$. In view of the numerous simplifications adopted in the theoretical model and of lack of data for the various coefficients, the results can be considered as satisfactory.

It is emphasized that the variance of $\ln \theta$ is equal to that of $\xi$ in our simplified model, according to (3.8). However, the inferred $\sigma_{\xi}=0.283$ (3.17) from the transport model is much larger than $\sigma_{\ln \theta}=0.068$ (3.3) inferred from measurements of $\theta$. This large discrepancy is attributed at present to the insufficient sampling of $\theta$ on one hand and on the neglection of additional sources of variability of the velocity, e.g. variability of $\alpha$, presence $\theta_{i r}$ and three-dimensional effects, on the other. These conjectures have to be elucidated in future experiments.

(vi) Estimates of coefficient of variation of mass arrival.

Since measurements of $K_{S}$ which could permit one to infer its spatial structure were not available, we have proceeded like in Sect. 2 and evaluated $C_{M}$ in unsaturated transport for various ratios between the caisson diameter $d$ and the logvelocity integral scale $I$. This time we used the variance $\sigma \xi=0.08$ of (3. 17) in the theoretical expression (3.14). The results of these computations are presented in Fig. 10 for $C_{M}$ as function of $\tau$ and for different $d^{\prime}=d / 1$. In Fig. 11 we present $\langle M\rangle / M_{0}$ and bounding curves for $95 \%$ interval of confidence, where $\langle M\rangle / M_{0}$ is 
given by (3.13). Finally, in Fig. 12 we represent the product $\mathrm{CV}_{\mathrm{NA}^{\prime 2}}$ which does not depend on $d^{\prime}$ for $d^{\prime} \gg 1$ (see Sect. 2). Fig. 11 shows clearly that the uncertainty of $M$ is much smaller than the corresponding one for saturated flow (Fig. 2), due to the smaller logvelocity variance. The uncertainty is quite small even if $d^{\prime}=40$, i.e. $I \simeq 7.5 \mathrm{~cm}$ and will be even smaller if threedimensional effects are accounted for.

\section{Summary and discussion of results. Recommendations for future experiments.}

The aim of the analysis of the caisson experiments of transport of a conservative solute in saturated and unsaturated experiments was to identify the spatially variable properties of the medium which led to the enhanced spreading of the solute as compared to that resulting from pore-scale dispersion. Toward this aim we have adopted a simple transport model ${ }^{9}$, motivated by the observed linear growth of the equivalent dispersivity with depth ${ }^{4,6,7}$. The basic transport model ${ }^{9}$, which approximates the actual stremtubes by a bundle of vertical columns and assumes heterogeneity in the horizontal plane only, has been recently found to be quite accurate for transport in the upper soil layer ${ }^{14}$. Generally, the experimental verification and validation of the model requires the independent measurement of the spatial distribution of permeability and of solute concentration at a large number of points and times. In the caisson saturated flow experiments only concentration measurements were available. Hence, the model has been applied in an inverse approach, namely by identifying the heterogeneous structure from solute mass arrival at different cross-sections. The model performed well6,7 in depicting the mass arrival curves, unlike the one assuming a constant effective dispersivity. In the present study (Sect. 2) we have examined the impact of the ratio between the caisson diameter and the permeability correlation scale in the horizontal plane on the variance of the mass arrival. The main conclusion is that this ratio has to be larger than say 100 to warrant the ergodic assumption, i.e. the approximate equality between the mass expected value and its one realization value.

The same vertical columns model ${ }^{9}$ has been applied to transport in the unsaturated flow experiments. This has been supplemented by a simplified gravitational flow model and has used the results of the saturated flow analysis. A fair agreement was found between parameters identified by the inverse procedure applied to the effluent concentration and those measured (mean velocity) or inferred (logconductivity variance).

One of the striking results of the experiments and analysis was the much smaller dispersion in the unsaturated flow than in the saturated one. Indeed, the logvelocity standard deviation was approximately 0.28 in the first and 1.2 in the latter. This difference for the same medium is 
clearly explained by the model: in the saturated flow experiments the boundary condition was of constant heads applied at the caisson ends and the velocity in our model was proportional to the saturated hydraulic conductivity which has the above large variance; in contrast, in the unsaturated flow experiments the boundary conditionat the top was of applied constant flux which was preserved all over the caisson in our model, the only source of velocity variability being the change of the moisture content in space. The variation of the latter with the saturated conductivity is much weaker. This experimental finding corroborated by a theretical model has far-reaching implications for field applications in similar conditions.

Future caisson experiments should comprise a thorough scanning of the permeability point values throughout the space in order to characterize its spatial structure independently of transport. As a starting point, the number of stations and their distribution should be based on the need to estimate with a small error the mean, variance and integral scales of logpermeability, assuming that the medium will have the same logpermeability variance as in the former experiments.

In the same vein the number of stations for measuring solute breakthrough curves should be at least equal to that in the previous saturated flow experiments to permit estimating the mass arrival curves at a few cross-sections. This is particularly true for reactive solutes for which part of the mass is not recovered. The major role played by the effluent concentration should be accounted for by its careful monitoring, avoiding as much as possible the delay effect of the conical section at the bottom. Finally, in the case of unsaturated flow conditions there is a need for a better coverage of the spatial distribution of moisture contents, as well as for measuring the characteristic curves (moisture content-matric potential and moisture content-hydraulic conductivity) for a sufficiently large number of samples of the caisson material.

Future caisson experiments may offer an opportunity to test the accuracy of stochastic transport models under controlled conditions which are not generally available in the field on one hand and at a scale much larger than that of ordinary laboratory columns on the other. 


\section{References}

1. Nyhan, J. W., W. L. Polzer, E.H. Essington, E. Cokal, L. Lane, E. Lopez, E. Stallings and R. Walker, A joint DOE/NRC field study of tracer migration in the unsaturated zone, LA10575-MS, Los Alamos National Laboratory, 1986.

2. Fuentes, H. R., and W. L. Polzer, Interpretative analysis of date for solute transport in the unsaturated zone, NUREG/CR-4737, U. S. Nuclear Regulatory Commisssion, 1986.

3. Polzer, W. L., E. H. Essington, H. R. Fuentes and J. W. Nyhan, Compilation of field scale caisson data on solute transport in the unsaturated zone, NUREG/CR-4720, U. S. Nuclear Regulatory Commisssion, 1986.

4. Springer, E. P., and H. R. Fuentes, Editors, Modelling study of the solute transport in the unsaturated zone, Workshop Proceedings, NUREG/CR4615, LA-10730-MS, Vol. 2, Los Alamos National Laboratory, 1987.

5. Fuentes, H. R. W. L. Polzer and E. P. Springer, Effects of influent boundary conditions on tracer migration and spatial variability features in intermediate-scale experiments, NUREG/CR4901, U. S. Nuclear Regulatory Commisssion, 1987.

6. Dagan, G., V. V. Nguyen and E. P. Springer, Analysis of transport in the upper soil layer and interpretation of the caisson experiments, Focus 89. Nuclear waste isolation in the unsaturated zone, Las Vegas, 1989.

7. Nguyen, V. V., G. Dagan, and E. P. Springer, Analysis of caisson transport experiments by travel time approach, in "Field-Scale Solute and Water Transport through Soil" K. Roth, H. Fluehler, W. A. Jury and J. C. Parker Eds., Birkhauser Verlag, Basel, 1991.

8. Dagan, G., Flow and Transport in Porous Formations, Springer-Verlag, 465 p., 1989.

9. Dagan, G. and E. Bresler, Solute dispersion in unsaturated heterogeneous soil at field scale I: Theory, Soil Science Soc. of Am. Journ., 43(3), 461-467, 1979.

10. Abramowitz, M., and I.A. Stegun, Handbook of Mathematical Functions, Dover, New York, 
1965.

11. Brooks, R. H. and A. T. Corey, Hydraulic properties of porous media, Hydrol Paper No. 3, Colorado State Univ., Fort Collins Colorado, 1964.

12. Abeele, W. V., Hydraulic testing of crushed Bandelier tuff, LA-10037-MS, Los Alamos National Lab., 1984.

13. Dullien, F. A. L., Porous Media : Fluid Transport and Structure, Academic Press, New York, 1984.

14. Protopapas, A. L. and R. L. Bras, The one-dimensional approximation for infiltration in heterogeneous soils, Water Resour. Res., 27, 1019-1027, 1991. 
Figure captions

Fig. 1 The coefficient of variation $C V_{M}=\left[\left\langle M^{2}\right\rangle /\langle M\rangle^{2}-1\right]$ (Eq. 2.10) of the solute mass arrival as function of the dimesionless time $r=t\langle u\rangle / z$ and for different $d^{\prime}=d / I$ (caisson diamter/logvelocity integral scale) under the saturated flow conditions.

Fig. 2 Like Fig. 1 for the expected value $\langle\mathrm{M}\rangle / \mathrm{M}_{0}$ (Eq. 2.9, solid line) and bounding curves at $95 \%$ interval of confidence of the solute mass arrival

Fig. 3 Like Fig. 1 for the product $\mathrm{CV}_{M}^{2} \cdot \mathrm{d}^{\prime 2}$.

Fig. 4 The cumulative effluent water volume $\left(10^{-3}\right.$ litre) as function of the elapsed time (days) in the first iodide experiment in unsaturated flow.

Fig. 5 The cumulative effluent water volume $\left(10^{-3}\right.$ litre) as function of the elapsed time (days) in the second iodide experiment in unsaturated flow.

Fig. 6 The cumulative effluent iodide mass (mg) as function of time (days) in the first iodide experiment in unsaturated flow.

Fig. 7 The cumulative effluent iodide mass (mg) as function of time (days) in the second iodide experiment in unsaturated flow.

Fig. 8 The normalized (by the total mass) cumulative effluent iodide mass in the two transport experiments as function of time (day) from the beginning of the solute pulse at the caisson top.

Fig. 9 Same as in Fig. 7 (solid lines) and the theoretical solution (eq. 3.13, dashed line).

Fig. 10 The coefficient of variation $C V_{M}=\left[\left(M^{2}\right) /(M\rangle^{2}-1\right]$ (Eq. 3.16) of the solute mass arrival as function of the dimesionless time $r=t(u) / z$ and for different $d^{\prime}=d / I$ (caisson diameter/logvelocity integral scale) under the unsaturated flow conditions. 
Fig. 11 Like Fig. 10 for the expected value $(M) / M_{0}$ (Eq. 3.13, solid line) and bounding curves at $95 \%$ interval of confidence of the solute mass arrival

Fig. 12 Like Fig. 10 for the product $\mathrm{CV}_{\mathrm{M}}^{2} \mathrm{~d}^{22}$. 


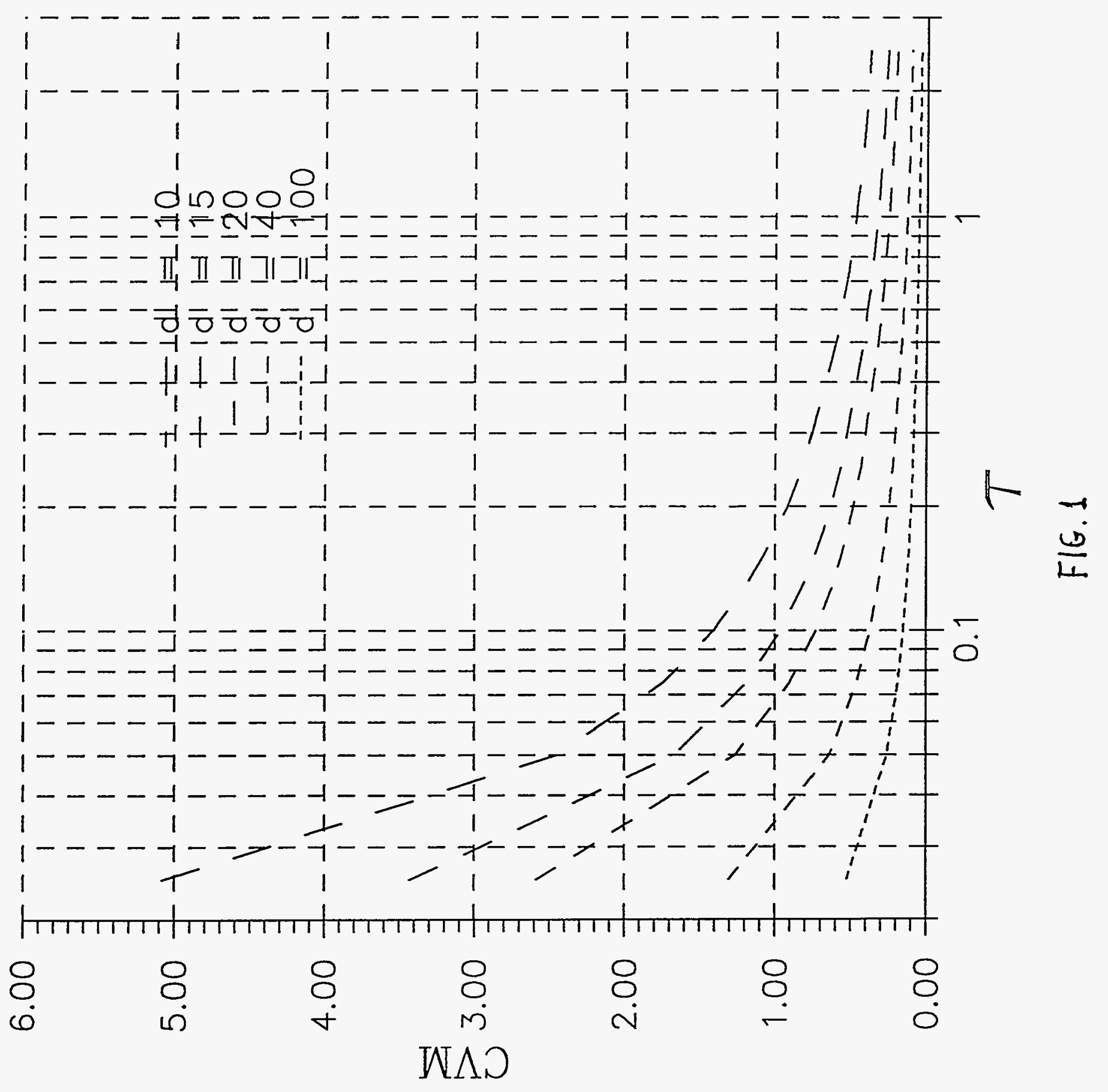




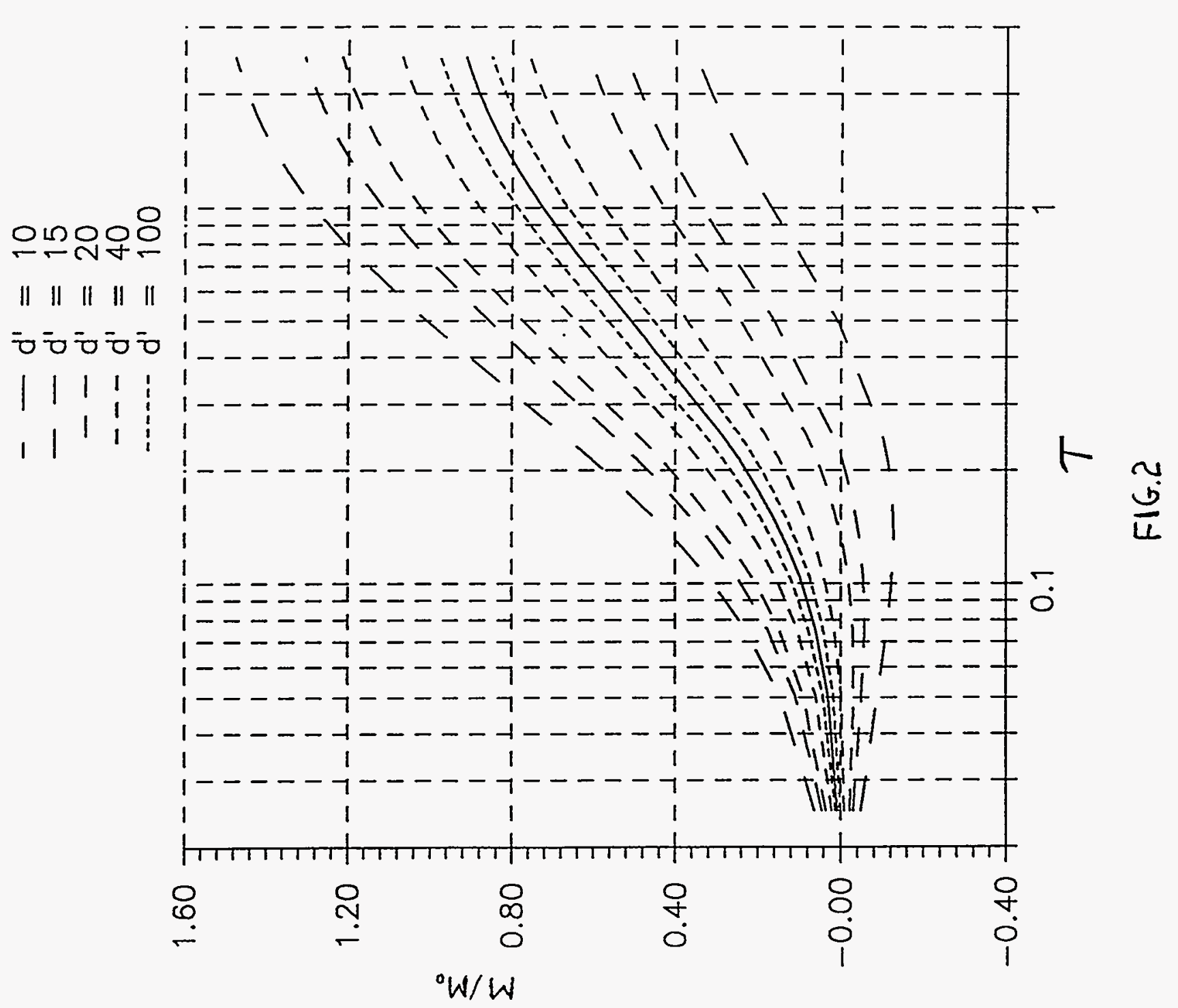




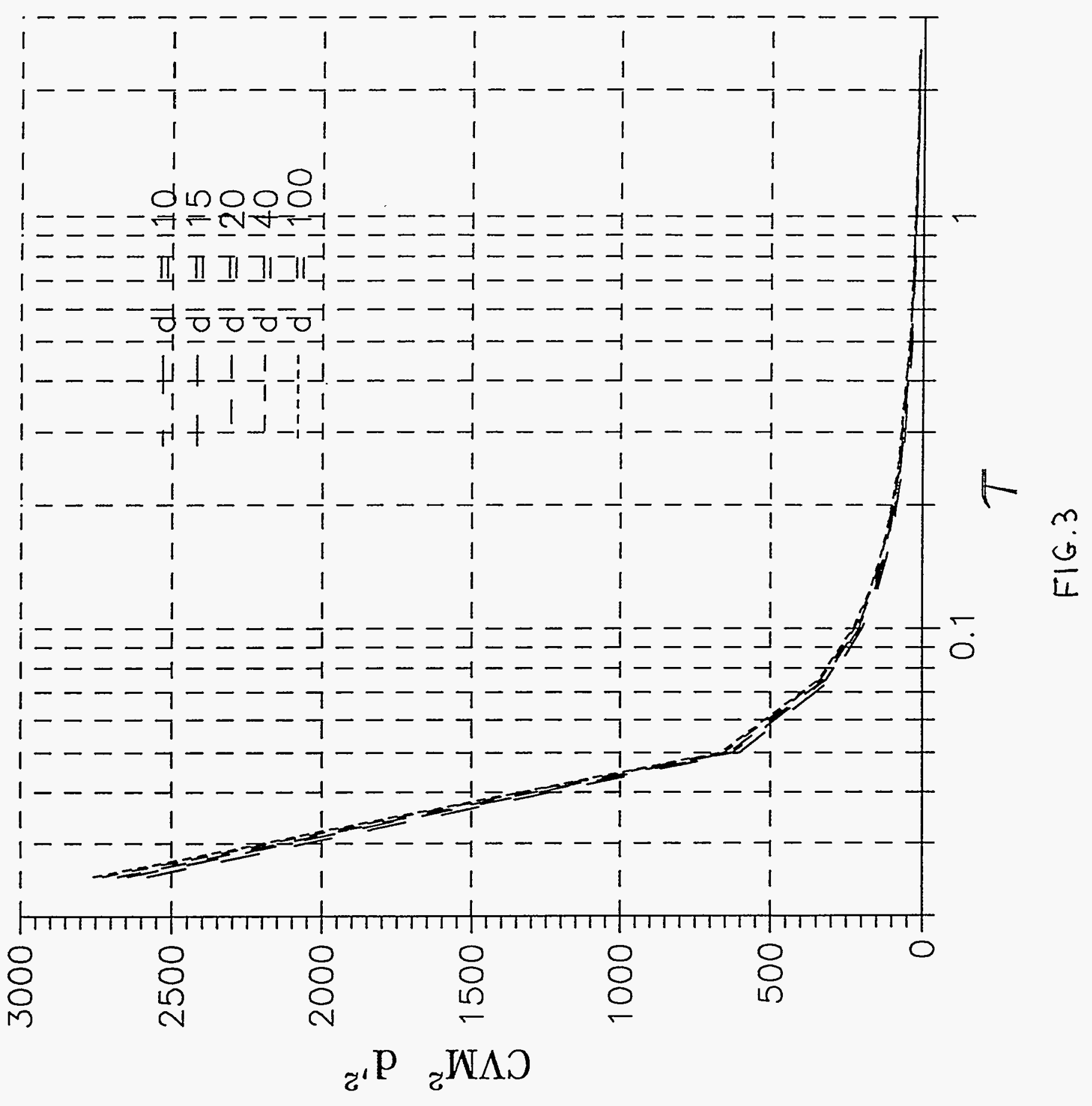




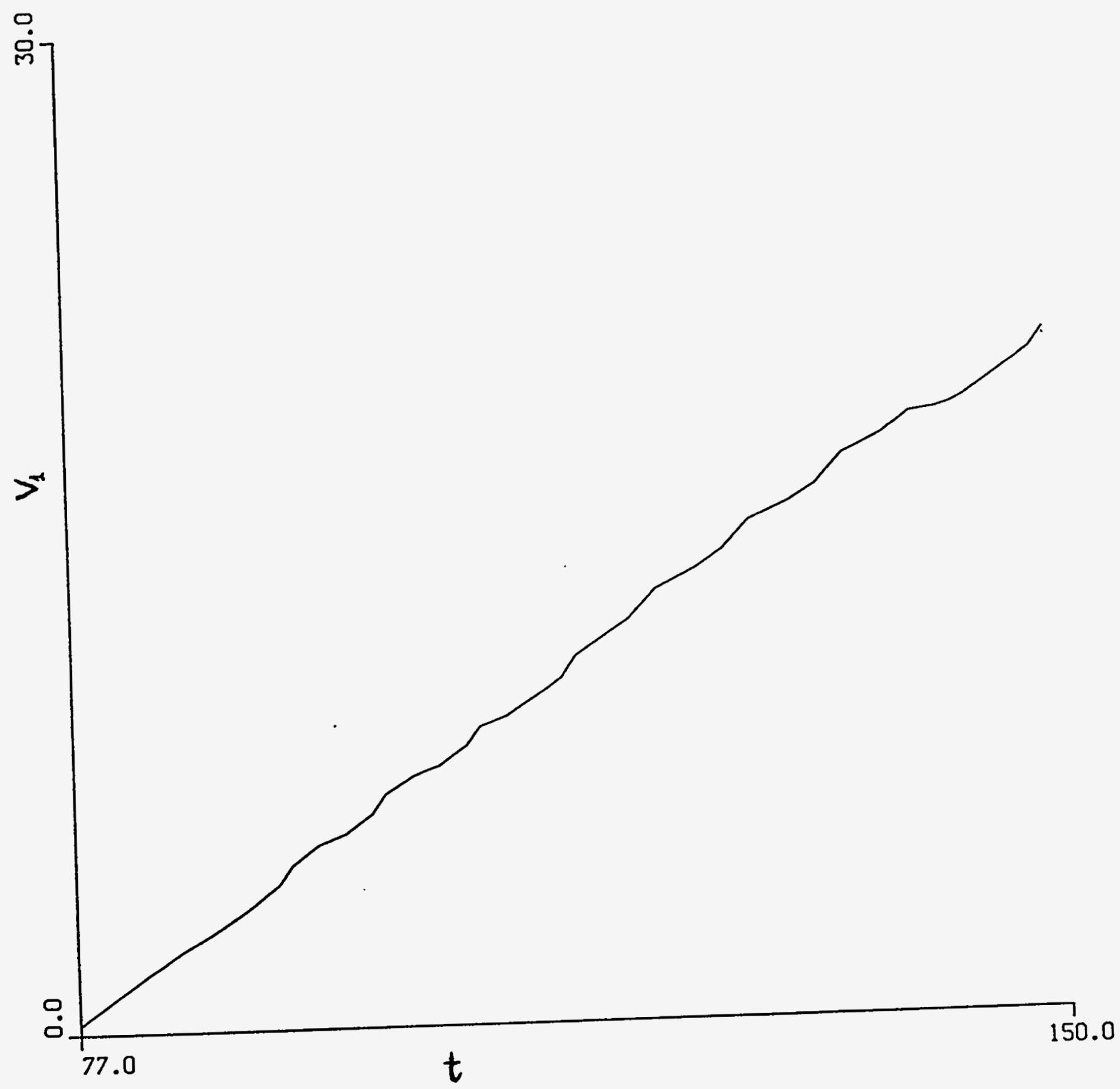

$F(G .4$ 


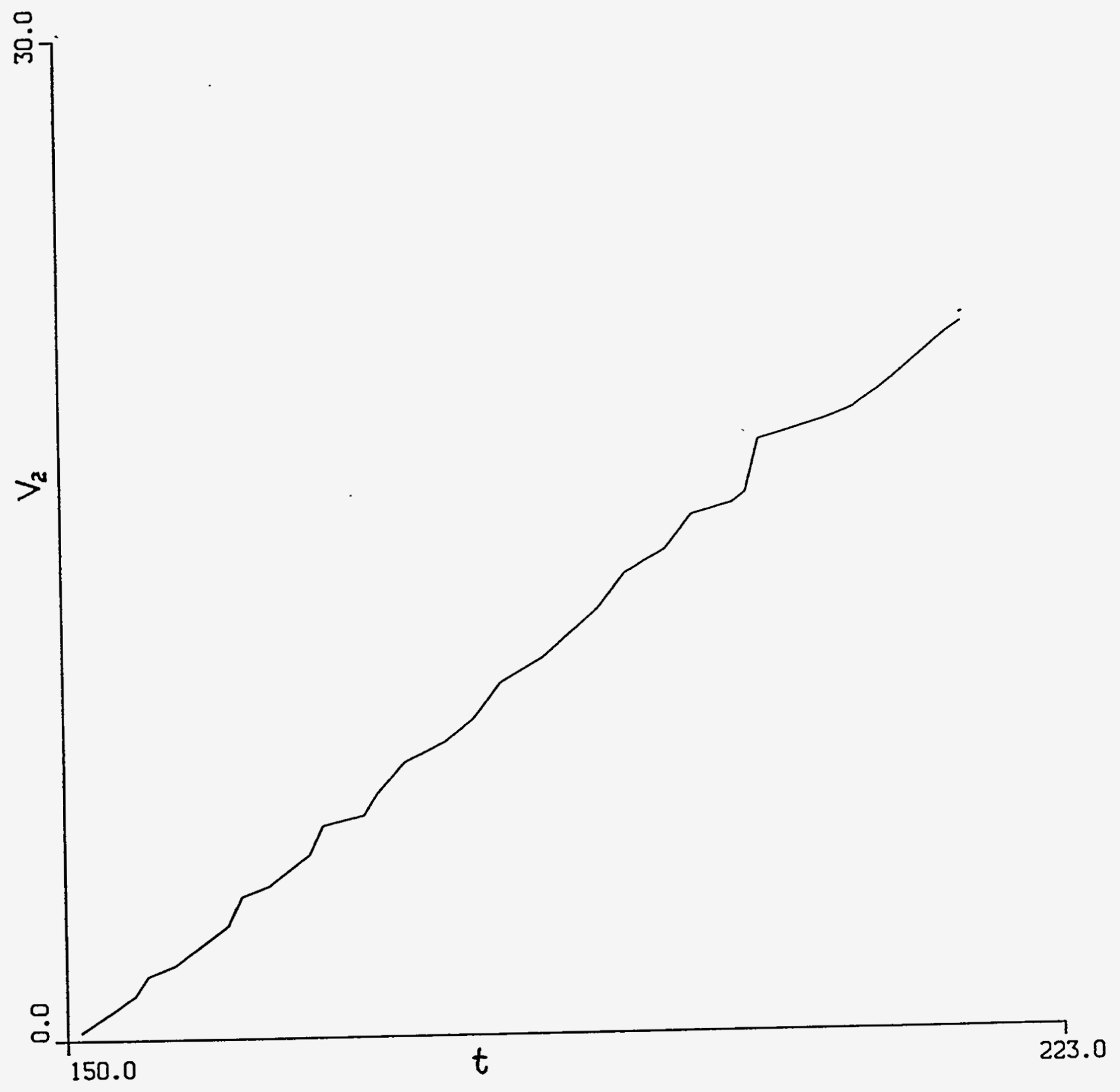

FIG. 5 


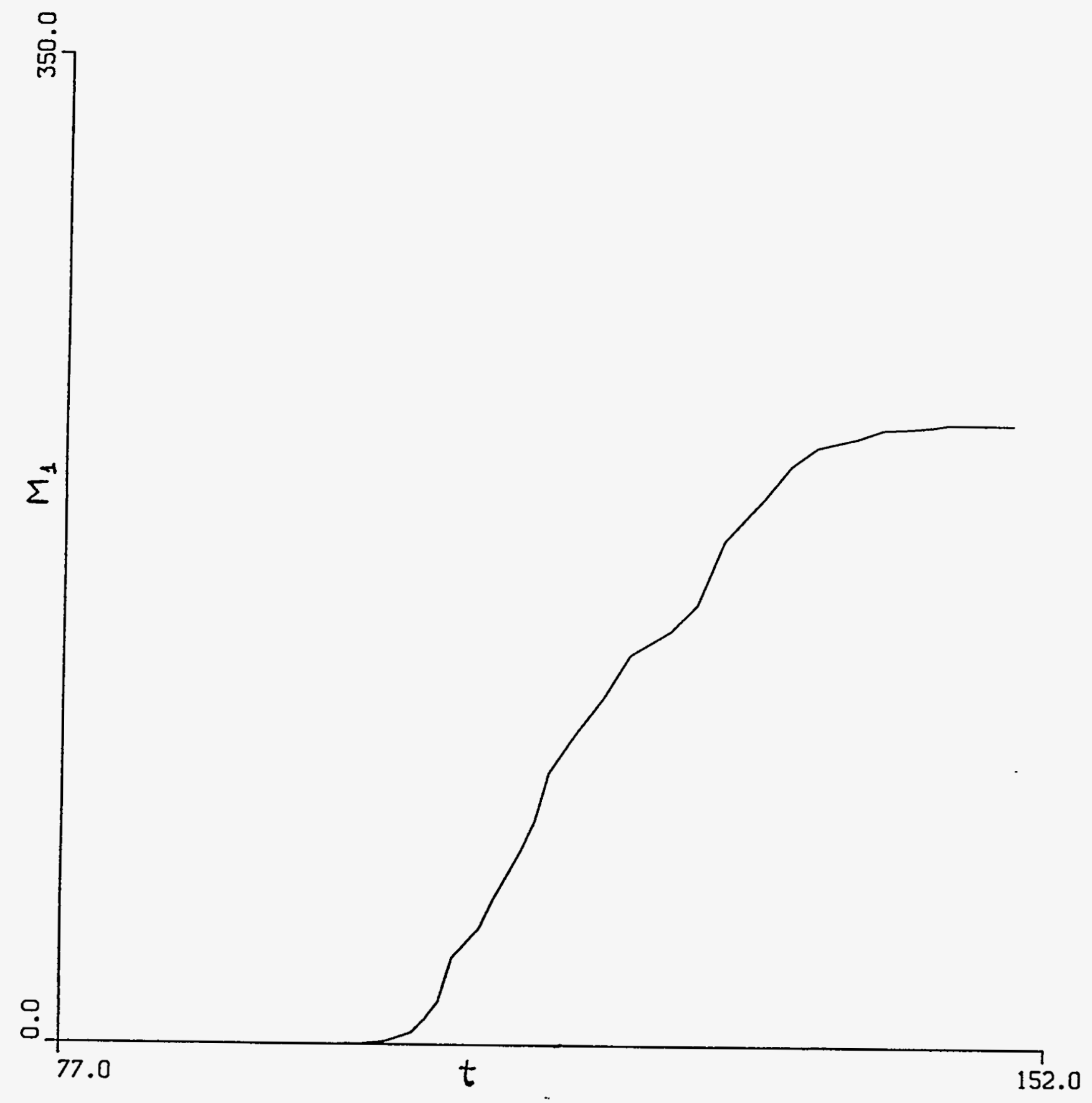

FIG. 6 


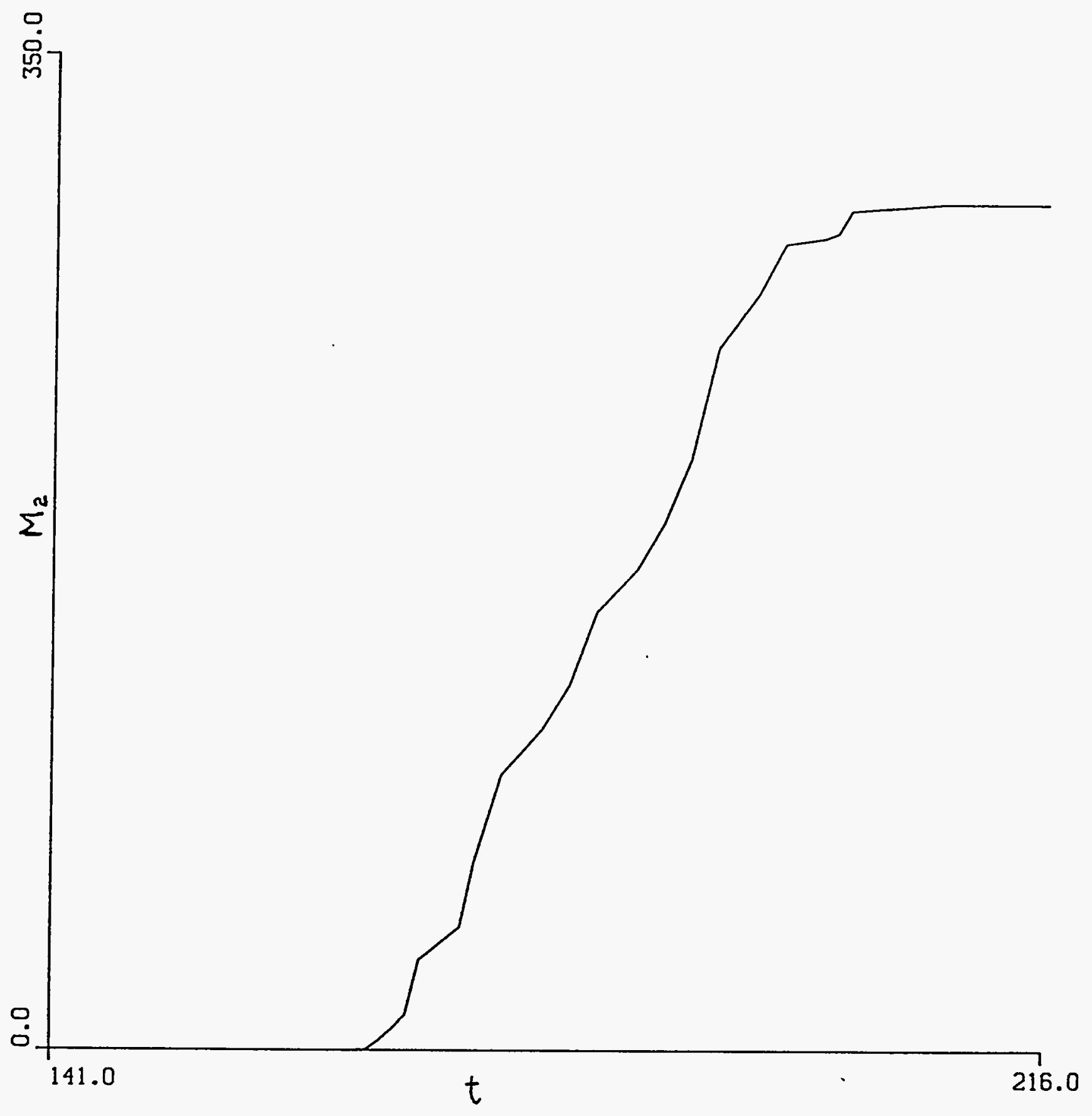

FIG. 7 


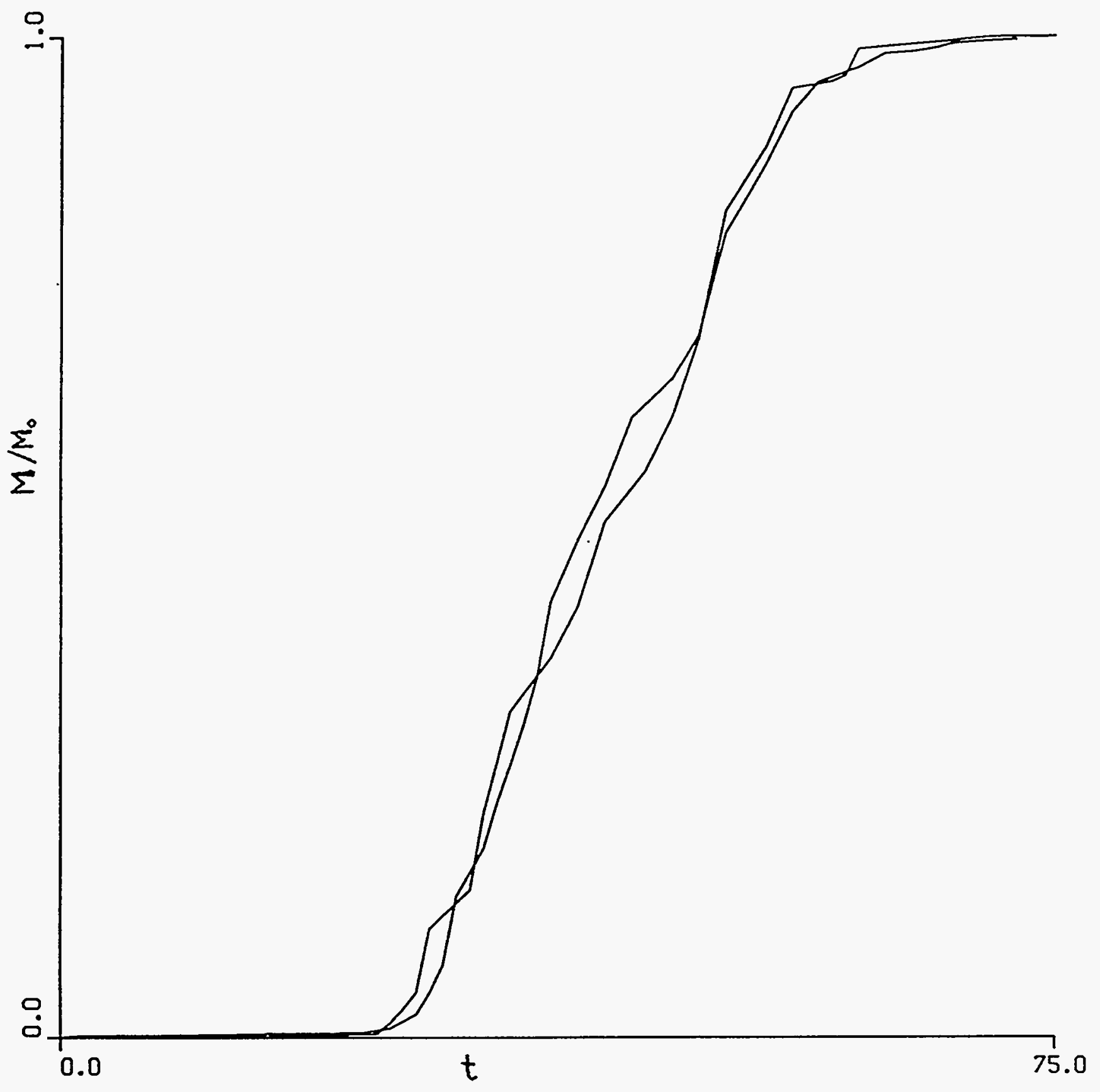

FIG.8 


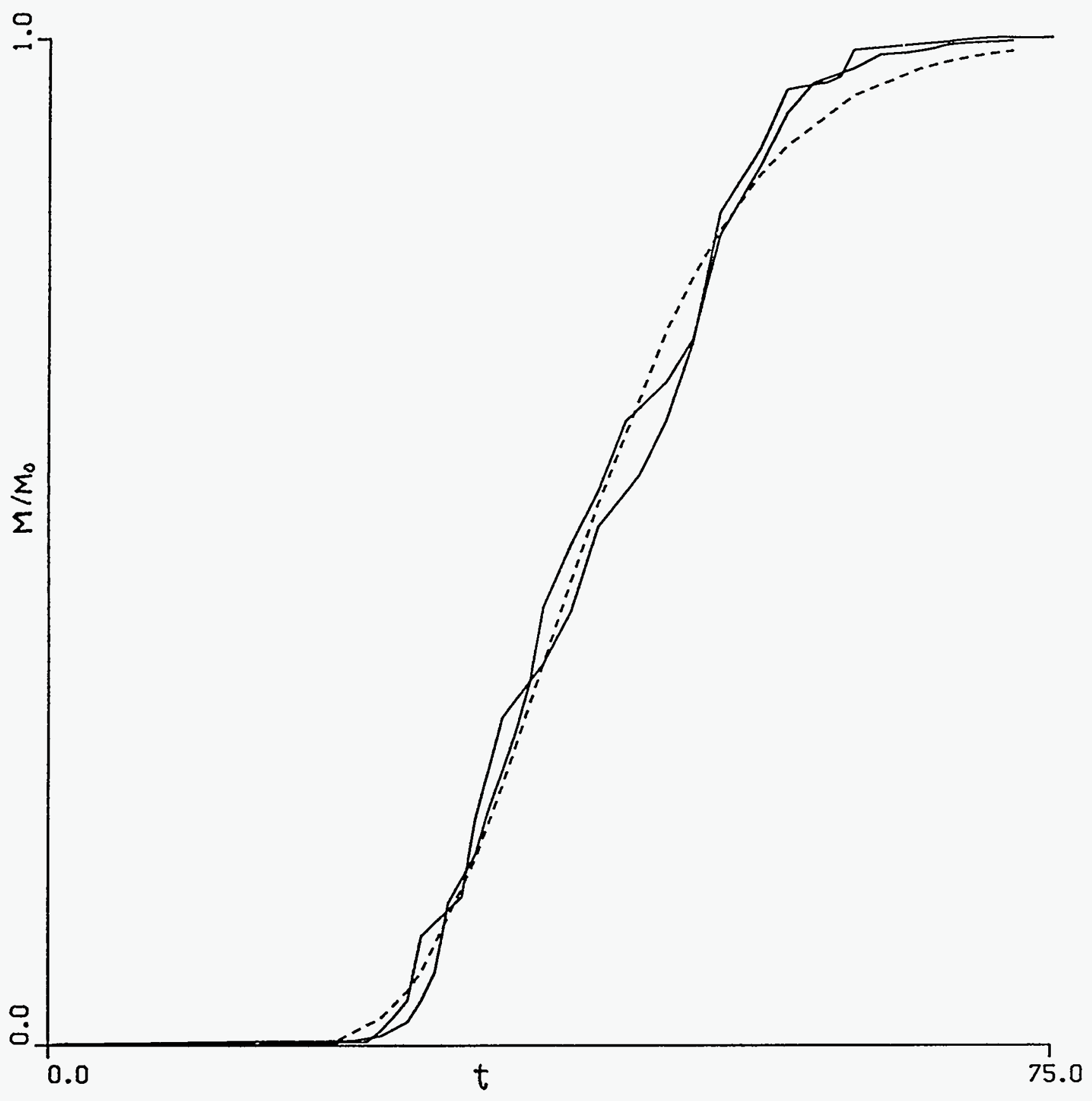

FIG. 9 


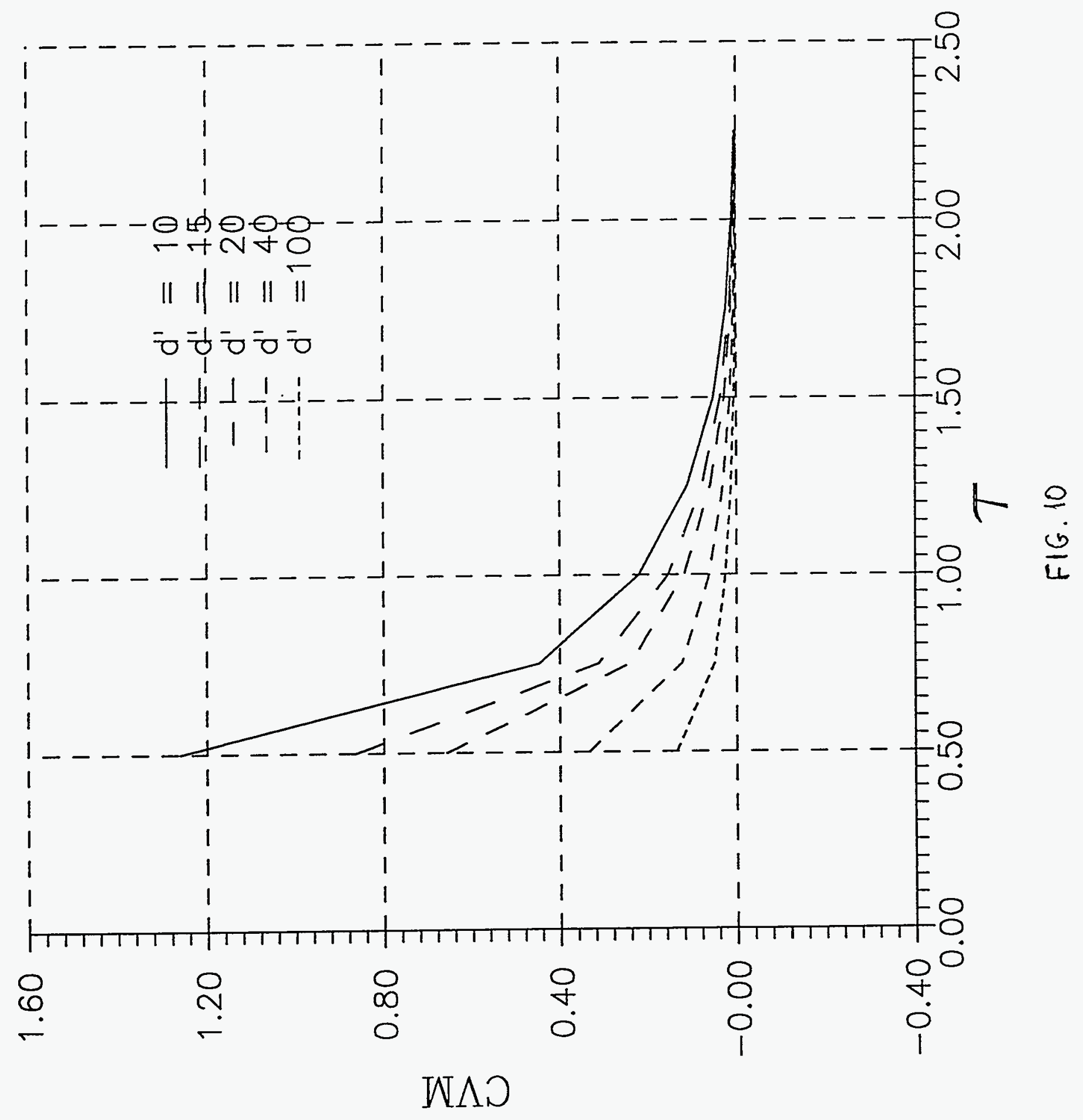




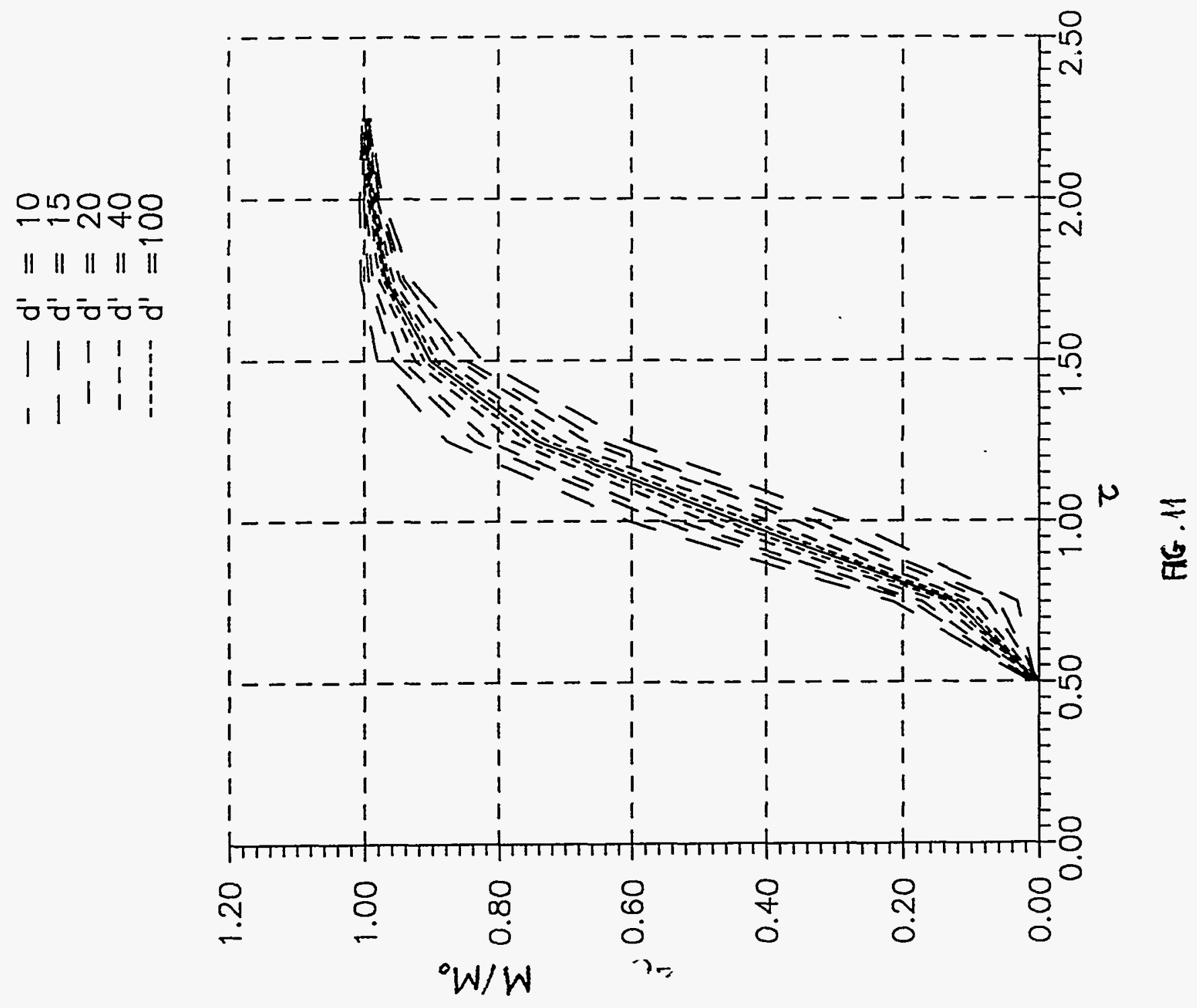




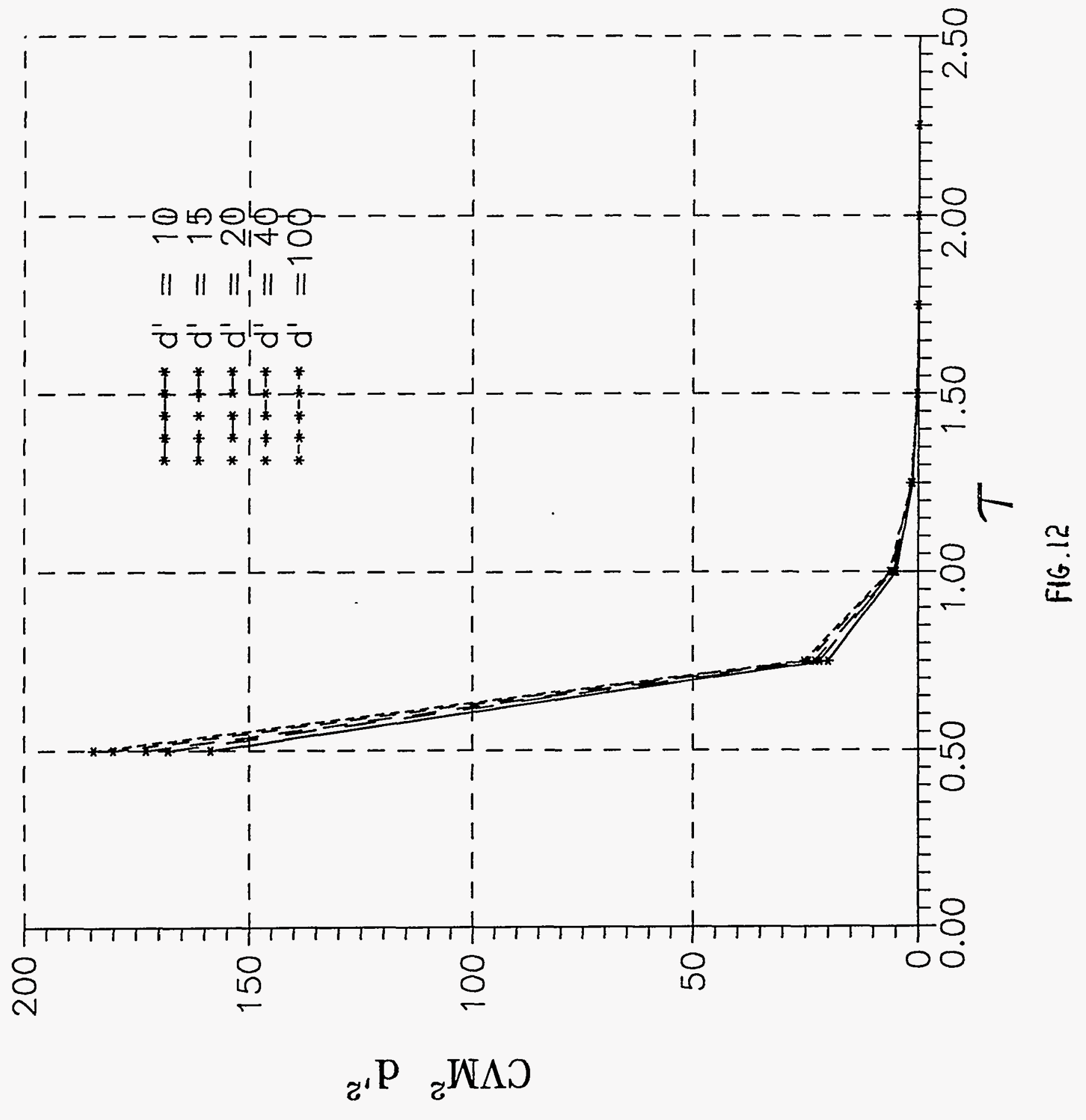

